\title{
PRODUCTS OF SEQUENTIALLY COMPACT SPACES AND THE V-PROCESS
}

BY

\author{
M. RAJAGOPALAN $\left({ }^{1}\right)$ AND R. GRANT WOODS $\left({ }^{2}\right)$
}

\begin{abstract}
In this paper we produce a family of sequentially compact, locally compact, $T_{2}$ first countable, scattered and separable spaces whose product is not countably compact and thus answer a problem of $C$. T. Scarborough and A. H. Stone [11] in the negative. We do this using the continuum hypothesis. We also produce a completely regular, $T_{2}$, sequentially compact space $K$ which is not $p$-compact for any $p \in \beta N-N$.
\end{abstract}

Notation 1.1. $N$ denotes the discrete space of integers $\{1,2,3, \ldots\}$. All spaces in this paper are Hausdorff. If $X$ is a topological space and $\pi$ is a partition of $X$ then $X / \pi$ denotes both the quotient set and quotient space. We use the continuum hypothesis $(\mathrm{CH})$ throughout. $\Omega$ denotes the first uncountable ordinal. We reserve greek symbols like $\alpha, \beta, \ldots$, etc. to denote a countable ordinal. English letters like $m, n$ are usually members of $N$. $\beta N$ denotes the Stone-Čech compactification of $N$, and if $X \subset \beta N$ then we give $X$ its subspace topology. Notions about filters and $\beta N$ can be found in [4]. We follow [5] for notions about scattered spaces. Hereafter, an element $P \in \beta N-N$ is considered to be given in advance.

Definition 1.2. Let $Y$ be an open subset of $\beta N, P \in \beta N-N$ and $\pi$ a partition of $Y$. We say that the pair $(Y, \pi)$ satisfies the condition $\mathrm{V}$ if the following hold:

(i) $P \notin Y$.

(ii) $N \subset Y$ and $\{n\} \in \pi$ for all $n \in N$.

(iii) $\pi$ is a partition of $Y$ by compact sets of $\beta N$.

(iv) $Y / \pi$ is countable, locally compact and $T_{2}$.

(v) Given a member $A \in \pi$ there is a compact open set $V$ of $\beta N$ so that $A \subset V \subset Y$ and $V$ is saturated under $\pi$.

Presented to the Society, January 14, 1975; received by the editors June 16,1975 and, in revised form, March 10, 1976.

AMS (MOS) subject classifications (1970). Primary 54C45, 54D45, 54D30, 54B10.

$K e y$ words and phrases. Scattered spaces, $p$-compact, countably compact, sequentially compact, $\beta N$, quotients, partitions.

(1) This author had a Memphis State University faculty research grant when this paper was written in the Summer of 1975.

(2) This author acknowledges support from the National Research Council of Canada.

- American Mathematical Society 1977 
Lemma 1.3. Let $(Y, \pi)$ be as in Definition 1.2 and satisfy V. Let $F_{1}$, $F_{2}, \ldots, F_{n}, \ldots$ be a sequence of distinct members of $\pi$ such that

$$
\left(\overline{\bigcup_{n=1}^{\infty} F_{n}}-\bigcup_{n=1}^{\infty} F_{n}\right) \cap Y=\varnothing \text { and } F=\overline{\bigcup_{n=1}^{\infty} F_{n}}-\bigcup_{n=1}^{\infty} F_{n} .
$$

Let $P \notin F$. Then there exists a compact, open set $W$ of $\beta N$ so that $P \notin W$ and $W \supset F$ and $W \cap Y$ is saturated under $\pi$.

Proof. From (iv) and (v) of Definition 1.2 we get that $Y$ can be expressed as a pairwise disjoint union $\cup_{n=1}^{\infty} M_{n}$ of compact, open sets $M_{1}$, $M_{2}, \ldots, M_{n}, \ldots$ of $\beta N$ so that $M_{n}$ is saturated under $\pi$ for all $n \in N$. If we put $M_{n} / \pi=A_{n}$ for all $n \in N$ then $Y / \pi$ is the disjoint union $\cup_{n=1}^{\infty} A_{n}$ of compact, open sets $A_{1}, A_{2}, \ldots, A_{n}, \ldots$ of $Y / \pi$. Let $q: Y \rightarrow Y / \pi$ be the canonical quotient map. Then the fact that $M_{n} / \pi$ is $T_{2}$ (from (iv) of Definition 1.2) gives that the restriction of $q$ to $M_{n}$ is closed. Hence $q$ : $Y \rightarrow Y / \pi$ is a closed map. Thus if $B \subset Y$ is open and $C \subset B$ is the union of all members of $\pi$ contained in $B$ then $C$ is open in $Y$ and hence in $\beta N$. Now $\cup_{n=1}^{\infty} F_{n}=\left(\cup_{n=1}^{\infty} F_{n}\right) \cup F$ and $F \cap Y=\varnothing$. So

$$
\left(\overline{\bigcup_{n=1}^{\infty} F_{n}}\right) \cap M_{k}=\left(\bigcup_{n=1}^{\infty} F_{n} \cap M_{k}\right)
$$

is compact for all $k \in N$. Put $\left(\cup_{n=1}^{\infty} F_{n}\right) \cap M_{k}=D_{k}$ for $k \in N$. Since $P \notin F$ and $F$ is closed we have that there is a compact, open set $S$ of $\beta N$ so that $F \subset S$ and $P \notin S$. Now

$$
\bigcup_{k=1}^{\infty} D_{k} \cup F=\bigcup_{n=1}^{\infty} F_{n} \cup F=\overline{\bigcup_{n=1}^{\infty} F_{n}}
$$

is compact and the collection $\left\{S, M_{1}, M_{2}, \ldots, M_{n}, \ldots\right\}$ is an open cover for $\cup_{n=1}^{\infty} F_{n}$. So there is an integer $k \geqslant 1$ so that $D_{l} \subset S$ if $l>k$ and $l \in N$. It is now clear that $\overline{\cup_{i=k+1}^{\infty} D_{i}} \supset F$. Using the closedness of the quotient map $q$ : $Y \rightarrow Y / \pi$ we get that the union $V$ of all members of $\pi$ contained in $Y \cap S \cap\left(\cup_{i=k+1}^{\infty} M_{i}\right)$ is open in $\beta N$. It follows from (iv) and (v) of Definition 1.2 that given $l>k$ and $l \in N$ there is a compact open set $V_{l}$ of $\beta N$ so that $D_{l} \subset V_{l} \subset M_{l} \cap V$ and $V_{l}$ is saturated under $\pi$ since $D_{l}$ is compact. Put $L=\bigcup_{l=k+1}^{\infty} V_{l}$. Then $L$ is an open set of $\underline{\beta} N$ so that $\bigcup_{i=k+1}^{\infty} D_{i} \subset L \subset S \cap$ $Y$ and $L$ is saturated under $\pi$. Then $\bar{L} \supset F$ and $\bar{L} \subset S$ and $\bar{L} \cap Y$ is saturated under $\pi$. Then $\bar{L}=W$ is the required open set of the theorem.

COROLlaRY 1.4. Let $Y, \pi$ be as in Lemma 1.3 and $(Y, \pi)$ satisfy the condition V. Let $F_{1}, F_{2}, \ldots, F_{n}, \ldots$ be a sequence of distinct members of $\pi$ so that $\bigcup_{n=1}^{\infty} F_{n}-\cup_{n=1}^{\infty} F_{n}=F$ is disjoint with $Y$ and $P \notin F$. Then there exists an 
open set $Y_{0}$ and a partition $\pi_{0}$ of $Y_{0}$ so that the following hold:

(a) $\left(Y_{0}, \pi_{0}\right)$ satisfies $\mathrm{V}$.

(b) $Y_{0} \supset F$.

(c) $Y_{0} \supset Y$ and $\pi_{0} \supset \pi$.

Proof. Let $W$ be as in the conclusion of Lemma 1.3. Let $Y_{0}=Y \cup W$, $\pi_{0}=\pi \cup\{W-Y\}$ if $F \neq \varnothing$ and $Y_{0}=Y, \pi_{0}=\pi$ otherwise. Then we get the corollary.

LEMMA 1.5. Let $Y_{n} \subset \beta N$ be an open set, $\pi_{n}$ a partition of $Y_{n}$ so that $\left(Y_{n}, \pi_{n}\right)$ satisfies $\mathrm{V}$, and $Y_{n+1} \supset Y_{n}$ and $\pi_{n+1} \supset \pi_{n}$ for $n \in N$. Let $Y=\cup_{n=1}^{\infty} Y_{n}$ and $\pi=\cup_{n=1}^{\infty} \pi_{n}$. Then $(Y, \pi)$ satisfies $\mathrm{V}$.

Proof. Easy to see.

COROLlaRy 1.6. Let $(Y, \pi)$ be as in Lemma 1.3 and satisfy condition V. Let $\mathcal{F}$ be a countable family of subsets of $\beta N$ so that if $A \in \mathcal{F}$ then there is a countable collection $\left\{G_{1}, G_{2}, \ldots, G_{n}, \ldots\right\}$ of distinct members of $\pi$ so that $A=\left(\cup_{n=1}^{\infty} G_{n}-\cup_{n=1}^{\infty} G_{n}\right)$ and $P \notin A$. Then there is an open set $Y_{0}$ of $\beta N$ and a paitition $\pi_{0}$ of $Y_{0}$ so that the following hold:

(a) $Y_{0} \supset Y$ and $\pi_{0} \supset \pi$.

(b) $\left(Y_{0}, \pi_{0}\right)$ satisfies the condition $\mathrm{V}$.

(c) If $A \in \mathcal{F}$ and $A \neq \varnothing$ then $Y_{0} \cap A \neq \varnothing$.

Proof. Write the members of $\mathscr{F}$ in a sequence $A_{1}, A_{2}, \ldots, A_{n}, \ldots$ If $Y \cap A_{1} \neq \varnothing$ or $A_{1}=\varnothing$ then put $Y_{1}=Y$ and $\pi_{1}=\pi$. If $Y \cap A_{1}=\varnothing$ then use Corollary 1.4 to get an open set $Y_{1}$ and a partition $\pi_{1}$ of $Y_{1}$ so that $Y_{1} \supset Y ; \pi_{1} \supset \pi ;\left(Y_{1}, \pi_{1}\right)$ satisfies $\mathrm{V}$ and $Y_{1} \supset A_{1}$. By induction suppose we have defined $Y_{i}$ and $\pi_{i}$ for $i=1,2, \ldots, n$ where $n \in N$. Assume further that the following hold:

(i) $Y \subset Y_{i} \subset Y_{j}$ for all $i, j=1,2, \ldots, n$ so that $i<j$.

(ii) $\pi \subset \pi_{i} \subset \pi_{j}$ for all $i, j=1,2, \ldots, n$ so that $i<j$.

(iii) $Y_{i} \cap A_{i} \neq \varnothing$ if $A_{i} \neq \varnothing$ where $i=1,2,3, \ldots, n$.

(iv) $\left(Y_{i}, \pi_{i}\right)$ satisfies condition $\mathrm{V}$ for all $i=1,2, \ldots, n$.

If $A_{n+1}=\varnothing$ or $A_{n+1} \cap Y_{n} \neq \varnothing$ then put $Y_{n+1}=Y_{n}$ and $\pi_{n+1}=\pi_{n}$. If $A_{n+1} \neq \varnothing$ and $A_{n+1} \cap Y_{n}=\varnothing$ then use Corollary 1.4 to get $\left(Y_{n+1}, \pi_{n+1}\right)$ so that $\left(Y_{n+1}, \pi_{n+1}\right)$ satisfies V; $Y_{n+1} \supset Y_{n} ; \pi_{n+1} \supset \pi_{n}$; and $Y_{n+1} \supset A_{n+1}$. Then the statements (i), (ii), (iii), (iv) above are true for $n+1$ also. Now put $Y_{0}=\cup_{n=1}^{\infty} Y_{n}$ and $\pi_{0}=\cup_{n=1}^{\infty} \pi_{n}$. Then this $\left(Y_{0}, \pi_{0}\right)$ satisfies the (a), (b), (c) of this corollary.

REMARK 1.7. Let $P \in \beta N-N$. We are going to give below a method for constructing an open set $Y$ of $\beta N$ and a partition $\pi$ of $Y$ by compact sets so that $P \notin Y$ and $Y / \pi$ is first countable, locally compact, $T_{2}$, locally count- 
able, $Y \supset N$ and $\{n\} \in \pi$ for all $n \in N$ and whenever $F_{1}, F_{2}, \ldots, F_{n}, \ldots$ is a sequence of distinct members of $\pi$ and $\left(\overline{\bigcup_{n=1}^{\infty} F_{n}}-\bigcup_{n=1}^{\infty} F_{n}\right)=F \neq \varnothing$ then $Y \cap F \neq \varnothing$. This method was introduced by the first author in [6] and was used in [7], and [9] to solve some problems of W. W. Comfort; Z. Semadeni; R. Telgarsky; P. Nyikos and S. P. Franklin.

Definition 1.8 (The V-PRocess). The following method of constructing an open set $Y$ of $\beta N$ and a partition $\pi$ of $Y$ is called the V-process. Put $Y_{1}=N$ and $\pi_{1}=\{\{n\} \mid n \in N\}$. Put $\mathscr{F}_{1}$ to be the collection of all sequences of distinct member of $\pi_{1}$. Put $\mathcal{G}_{1}$ to be the collection of all sets $A$ of the form

$$
A=\left(\bar{\bigcup}_{n=1}^{\infty} F_{n}-\bigcup_{n=1}^{\infty} F_{n}\right)
$$

where the sequence $\left(F_{1}, F_{2}, \ldots, F_{n}, \ldots\right)$ is a member of $F_{1}$ and $P \notin A$. Then $\mathcal{G}_{1}$ is uncountable. Let $\left\{A_{11}, A_{12}, \ldots, A_{1 \alpha}, \ldots\right\}$ be as well ordering of $\mathcal{G}_{1}$ (using $(\mathrm{CH})$ ) where $1 \leqslant \alpha<\Omega$. Now assume that $\theta \in[1, \Omega)$ is a successor ordinal equal to $\gamma+1$ and that we have defined $Y_{\alpha}, \pi_{\alpha}$ and $A_{\alpha \delta}$ for all $\alpha$ so that $1<\alpha \leqslant \gamma$ and $\delta$ so that $1 \leqslant \delta<\Omega$. Assume further that $\left(Y_{\alpha}, \pi_{\alpha}\right)$ satisfies the condition $\mathrm{V}$ for all $\alpha \in[1, \gamma]$ and $Y_{\alpha} \supset Y_{\beta}$ and $\pi_{\alpha} \supset \pi_{\beta}$ for all $\alpha$, $\beta$ in $[1, \gamma]$ and $\beta<\alpha$. Assume that given $\alpha \in[1, \gamma]$ and $\delta \in[1, \Omega)$ there is a sequence $F_{1}, F_{2}, \ldots, F_{n}, \ldots$ of distinct members of $\pi_{\alpha}$ so that $A_{\alpha \delta}=\overline{\bigcup_{n=1}^{\infty} F_{n}}-\bigcup_{n=1}^{\infty} F_{n}$ and $P \notin A_{\alpha \delta}$. Conversely assume that given $\alpha \in$ $[1, \gamma]$ and a sequence $G_{1}, G_{2}, \ldots, G_{n}, \ldots$ of distinct members of $\pi_{\alpha}$ so that $P \notin \overline{\cup_{n=1}^{\infty} G_{n}}$ there is an $\eta \in[1, \Omega)$ so that $A_{\alpha \eta}=\left(\overline{\cup_{n=1}^{\infty} G_{n}}-\bigcup_{n=1}^{\infty} G_{n}\right)$. Then using Corollary 1.6 construct an open set $Y_{\theta}=Y_{\gamma+1}$ and a partition $\pi_{\theta}=\pi_{\gamma+1}$ of $Y_{\gamma+1}$ so that the following conditions (a), (b), (c), (d) hold:

(a) $Y_{\gamma+1}$ is an open subset of $\beta N$ and $\pi_{\gamma+1}$ is a partition of $Y_{\gamma+1}$ by compact sets.

(b) $\left(Y_{\gamma+1}, \pi_{\gamma+1}\right)$ satisfies condition $\mathrm{V}$.

(c) $Y_{\gamma+1} \supset Y_{\gamma}$ and $\pi_{\gamma+1} \supset \pi_{\gamma}$.

(d) $Y_{\gamma+1} \cap A_{\eta \delta} \neq \varnothing$ provided $1 \leqslant \eta \leqslant \gamma$ and $1 \leqslant \delta \leqslant \omega^{\gamma}$ and $A_{\eta \delta} \neq \varnothing$. Now put $\mathscr{F}_{\gamma+1}$ to be the collection of all sequences $\left\{F_{1}, F_{2}, \ldots, F_{n}, \ldots\right\}$ of distinct members of $\pi_{\gamma+1}$ so that $P \notin \bigcup_{n=1}^{\infty} F_{n}$. Put $\mathcal{G}_{\gamma+1}$ to. be the collection of all sets of the form $A=\left(\bigcup_{n=1}^{\infty} F_{n}-\bigcup_{n=1}^{\infty} F_{n}\right)$ where the sequence $F_{1}$, $F_{2}, \ldots, F_{n}, \ldots$ is in $\mathscr{F}_{\gamma+1}$. We well order $\mathcal{G}_{\gamma+1}$ as $A_{(\gamma+1) 1}$, $A_{(\gamma+1) 2}, \ldots, A_{(\gamma+1) \delta}, \ldots$ with $\delta \in[1, \Omega)$ by using $(\mathrm{CH})$. (At this point we remark that it is easy to see that $\mathcal{G}_{\gamma+1}$ is uncountable and has cardinality $c$ and hence $\mathcal{G}_{\gamma+1}$ can be well ordered by $[1, \Omega)$.)

Now assume that $\theta$ is a limit ordinal in $[1, \Omega)$ and we have defined $Y_{\alpha}, \pi_{\alpha}$ and $A_{\alpha \delta}$ for all $\alpha, \delta$ where $\alpha \in[1, \theta)$ and $\delta \in[1, \Omega)$. Put $Y_{\theta}=\cup_{\alpha<\theta} Y_{\alpha}$ and $\pi_{\theta}=\bigcup_{\alpha<\theta} \pi_{\alpha}$. Let $F_{\theta}$ denote the collection of all sequences $\left(F_{1}\right.$, $\left.F_{2}, \ldots, F_{n}, \ldots\right)$ of distinct members of $\pi_{\theta}$ so that $P \notin \overline{\bigcup_{n=1}^{\infty} F_{n}}$. Let $\mathcal{G}_{\theta}$ be 
the collection of all sets $A$ of the form $\overline{\cup_{n=1}^{\infty} F_{n}}-\cup_{n=1}^{\infty} F_{n}$ where the sequence $\left(F_{1}, F_{2}, \ldots, F_{n}, \ldots\right)$ is in $\mathscr{F}_{\theta}$. Then $\mathcal{G}_{\theta}$ is uncountable. Well order $\mathcal{S}_{\theta}$ as $A_{\theta 1}, A_{\theta 2}, \ldots, A_{\theta \delta}, \ldots$ where $1 \leqslant \delta<\Omega$.

Finally put $Y_{p}=\cup_{\theta \in[1, \Omega)} Y_{\theta}$ and $\pi_{p}=\cup_{\theta \in[1, \Omega)} \pi_{\theta}$ and $X_{p}=Y_{p} / \pi_{p}$.

The $\mathrm{V}$-process ends here.

The spaces $X_{p}$ constructed above will be the spaces that will answer a problem of C. Scarborough and A. H. Stone [11]. We proceed below to show that $X_{p}$ is a first countable, locally compact, $T_{2}$ sequentially compact space for all $P \in \beta N-N$.

TheOREM 1.9. Let $P \in \beta N-N$, and let $Y_{p}, \pi_{p}, X_{p}, Y_{\alpha}, \pi_{\alpha}, A_{\alpha \delta}$ be as in the definition of V-process for all $\alpha \in[1, \Omega)$ and $\delta \in[1, \Omega)$. Then the space $X_{p}$ obtained in the V-process is locally compact, $T_{2}$, locally countable and hence first countable. Moreover if $F_{1}, F_{2}, \ldots, F_{n}, \ldots$ is a sequence of distinct members of $\pi_{p}$ and $\overline{\bigcup_{n=1}^{\infty} F_{n}}-\bigcup_{n=1}^{\infty} F_{n}=A \neq \varnothing$ then $A \cap Y_{p} \neq \varnothing$.

Proof. Now $Y_{\alpha}$ is open in $\beta N, Y_{\alpha} \supset Y_{\beta}$ and $\pi_{\alpha} \supset \pi_{\beta}$ for all $\alpha, \beta \in[1, \Omega)$ and $\alpha>\beta$. (These assertions are easy to see from the definition of $Y_{\alpha}$ and $\pi_{\alpha}$ in the V-process.) Put $X_{\alpha}=Y_{\alpha} / \pi_{\alpha}$ for all $\alpha \in[1, \Omega)$. Then $X_{\alpha} \subset X_{\beta}$ in a natural way and $X_{\alpha}$ is open in $X_{\beta}$ because $\pi_{\alpha} \subset \pi_{\beta}$ for all $\alpha, \beta \in[1, \Omega)$ and $\alpha<\beta$. Moreover, $X_{p}=\cup_{\alpha \in[1, \Omega)} X_{\alpha}$. Since $\left(Y_{\alpha}, \pi_{\alpha}\right)$ satisfies condition $\mathrm{V}$ we have that $X_{\alpha}$ is countable, locally compact, $T_{2}$ and hence first countable for all $\alpha \in[1, \Omega)$. Since $X_{p}=\cup_{\alpha \in[1, \Omega)} X_{\alpha}$ and $X_{\alpha}$ is open in $X_{p}$ for all $\alpha \in[1, \Omega)$ we get that $X_{p}$ is $T_{2}$, locally compact, locally countable and first countable. Now let $F_{1}, F_{2}, \ldots, F_{n}, \ldots$ be a sequence of distinct members of $\pi_{p}$ and let $\bigcup_{n=1}^{\infty} F_{n}-\cup_{n=1}^{\infty} F_{n}=A \neq \varnothing$. We claim that $A \cap Y_{p} \neq \varnothing$. To see this we first of all see from the definition of $\pi_{p}$ that given in $n \in N$ there is an $\alpha_{n} \in[1, \Omega)$ so that $F_{n} \in \pi_{\alpha_{n}}$. So, if $\alpha \in[1, \Omega)$ and $\alpha>\alpha_{n}$ for all $n \in N$ then $F_{n} \in \pi_{\alpha}$ for all $n \in N$. Now we have two cases.

Case (i). $P \notin \overline{\cup_{n=1}^{\infty} F_{n}}-\cup_{n=1}^{\infty} F_{n}=A$. Then there is a $\delta$ in $[1, \Omega)$ so that $A=A_{\alpha \delta}$. (This is from the definition of $A_{\alpha \delta}$ 's.) Now there is a $\gamma \in[1, \Omega)$ so that $\gamma>\alpha$ and $\delta<\omega^{\gamma}$. Then $Y_{\gamma} \cap A_{\alpha \delta} \neq \varnothing$ from the construction of $Y_{\gamma}$ in the V-process. So $Y_{p} \cap A_{\gamma \delta}$ is also $\neq \varnothing$.

Case (ii). $P \in A=\left(\bigcup_{n=1}^{\infty} F_{n}-\bigcup_{n=1}^{\infty} F_{n}\right)$. Then we claim that there is a subsequence $F_{n_{1}}, F_{n_{2}}, \ldots, F_{n_{1}}, \ldots$ of $F_{1}, F_{2}, \ldots, F_{n}, \ldots$ so that $P \notin B=\overline{\bigcup_{r=1}^{\infty} F_{n_{r}}}-\bigcup_{r=1}^{\infty} F_{n_{r}}$ and $B \neq \varnothing$, and $B \cap Y_{\alpha}=\varnothing$. If this is proved then the Case (i) aboe shows that $Y_{p} \cap B \neq \varnothing$ and $B \subset \overline{\cup_{n=1}^{\infty} F_{n}}$ $\bigcup_{n=1}^{\infty} F_{n}=A$. Thus we will get $Y_{p} \cap A \neq \varnothing$ in this case too. Now $\left(Y_{\alpha}, \pi_{\alpha}\right)$ satisfies condition V. So, as in the proof of Lemma 1.3 we can find pairwise disjoint compact, open subsets $M_{1}, M_{2}, \ldots, M_{n}, \ldots$ of $\beta N$ so that $Y_{\alpha}=$ $\cup_{n=1}^{\infty} M_{n}$ and each $M_{n}$ is saturated under $\pi_{\alpha}$. Since $P \in \overline{\cup_{n=1}^{\infty} F_{n}}$ we have that $\bigcup_{n=1}^{\infty} F_{n}$ is not compact. So $\cup_{n=1}^{\infty} F_{n}$ cannot be contained in a finite 
union of the $M_{n}$ 's. So there exists an infinite sequence $n_{1}<n_{2}<n_{3}$ $<\cdots<n_{k}<\ldots$ of integers in $N$ so that each $M_{n_{k}}$ contains at least one $F_{n}$. Now $\beta N$ is extremally disconnected. So $P$ can belong to the closure of at most one of the sets $\bigcup_{k=1}^{\infty} M_{n_{(2 k)}}$ or $\bigcup_{k=1}^{\infty} M_{n_{(2 k+1)}}$.

Choose the sequence $\left(M_{n_{2 k}}\right)$ if $P \notin \cup_{k=1}^{\infty} M_{n_{2 k}}$ and the sequence $\left(M_{n_{(2 k+11}}\right)$ otherwise. Rename the chosen sequence as $\left(M_{p_{k}}\right)$ for convenience. For each $k \in N$ choose one set $F_{r_{k}}$ from the sequence $\left(F_{n}\right)$ so that $F_{r_{k}} \subset M_{p_{k}}$ for all $k \in N$. Then $\cup_{k=1}^{\infty} F_{r_{k}}$ cannot be compact since the collection $\left\{M_{p_{k}} \mid k \in N\right\}$ is an open cover for $\cup_{k=1}^{\infty} F_{r_{k}}$ from which a finite cover cannot be extracted. So $\overline{\bigcup_{k=1}^{\infty} F_{r_{k}}}-\cup_{k=1}^{\infty} F_{r_{k}}=B \neq \varnothing$ and $P \notin B$. Clearly $B \cap Y_{\alpha}=\varnothing$ and hence $B \subset \cup_{n=1}^{\infty} F_{n}-Y_{\alpha} \subset \overline{\bigcup_{n=1}^{\infty} F_{n}}-\cup_{n=1}^{\infty} F_{n}=A$. So the subsequence $\left(F_{r_{k}}\right)$ is the required subsequence and we have the theorem.

THEOREM 1.10. Let $P \in \beta N-N$, and let $X_{p}$ be the space obtained by the V-process. Then $X_{p}$ is countably compact. Since it is also first countable, it is sequentially compact.

Proof. Let $Y_{p}, \pi_{p}, Y_{\alpha}, \pi_{\alpha}$ be as in the definition of V-process for all $\alpha$ in [1, $\Omega)$. Let $q: Y_{p} \rightarrow X_{p}$ be the canonical quotient map. Let $\left\{x_{1}, x_{2}, \ldots, x_{n}, \ldots\right\}$ be a countable discrete infinite set in $X_{p}$. Choose $F_{n} \in \pi_{p}$ so that $q\left(F_{n}\right)=x_{n}$ for all $n \in N$. Now $Y_{p} \cap A \neq \varnothing$ by Theorem 1.9 where $A$ $=\bigcup_{n=1}^{\infty} F_{n}-\bigcup_{n=1}^{\infty} F_{n}$. Let $y \in Y_{p} \cap A$. Then $q(y) \in X_{p}$ is clearly a cluster point of the set $\left\{x_{1}, x_{2}, \ldots, x_{n}, \ldots\right\}$. So $X_{p}$ is countably compact and Theorem 1.9 gives that $X_{p}$ is also sequentially compact.

Definition 1.11. Let $p \in \beta N-N$ be given. Let $X$ be a topological space. $X$ is called $p$-compact if given any 1-1 map $f: N \rightarrow X$ there is a continuous extension $g: N \cup\{p\} \rightarrow X$ of $f$ from $N \cup\{p\}$ into $X$.

REMARK. The idea of $p$-compactness was first introduced in a different form by A. R. Bernstein in [2].

Lemma 1.12. Let $p \in \beta N-N$ be given. Then the space $X_{p}$ obtained by the use of V-process is not p-compact. Moreover, $X_{p}$ is scattered. So given an element $p \in \beta N-N$, there is a scattered, locally compact, $T_{2}$, locally countable, first countable, sequentially compact, non p-compact space.

Proof. Let $X_{p} \cup\{\infty\}$ be the one point compactification of $X_{p}$. Let $q$ : $Y_{p} \rightarrow X_{p}$ the quotient map and $f=q \mid N$. Then $f: N \rightarrow X_{p}$ is a function which is one-to-one on $N$. We are going to show that this $f$ has no continuous extension from $N \cup\{p\} \rightarrow X_{p}$. Let $g: \beta N \rightarrow X \cup\{\infty\}$ be the function which maps $\beta N / Y_{p}$ onto $\{\infty\}$ and coincides with $q$ on $Y_{p}$. Then $g$ is continuous and is the unique continuous extension of $f$ to $\beta N$. In particular $g(p)=\infty$. Now $\beta(N \cup\{p\})=\beta N$ so every continuous function $\phi: N \cup\{p\} \rightarrow X_{p} \cup\{\infty\}$ must extend to a unique continuous function $\tilde{\phi}: \beta N \rightarrow X_{p} \cup\{\infty\}$. So if there 
is a continuous extension $\tilde{f}: N \cup\{p\} \rightarrow X_{p}$ of $f$ to $N \cup\{p\}$ then there will be a continuous extension $\tilde{g}$ of $\tilde{f}$ from $\beta N \rightarrow X_{p} \cup\{\infty\}$ and this $\tilde{g}$ will also be a continuous extension of $f$. Now $\tilde{g}(p) \in X_{p}$ and $g(p)=\infty$. So $\tilde{g} \neq g$. So, if an extension $\tilde{f}$ for $f$ exists as a continuous function from $N \cup\{p\} \rightarrow X_{p}$ then $f: N \rightarrow X_{p} \cup\{\infty\}$ will have two continuous extensions $g, \tilde{g}$ from $\beta N \rightarrow X_{p} \cup$ $\{\infty\}$ which is not possible. So $X_{p}$ is not $p$-compact. The second part of the lemma now follows easily from Theorem 1.10 .

Lemma 1.13. Let $X$ be a topological space, and let $f: N \rightarrow X$ a one-to-one function from $N$ into $X$. Then $f(N)$ has a cluster point in $X$ if and only if there is at least one $p_{0} \in \beta N-N$ so that $f$ extends continuously to a function $g$ : $N \cup\left\{p_{0}\right\} \rightarrow X$ from $N \cup\left\{p_{0}\right\}$ into $X$.

Proof. If $f$ has a continuous extension $\tilde{f}$ from $N \cup\left\{p_{0}\right\}$ into $X$ for some $p_{0} \in \beta N-N$ then $\tilde{f}\left(p_{0}\right)$ is a cluster point of $f(N)$. Conversely let $f(N)$ have a cluster point $l$ in $X$. Let $\mathfrak{V}$ be the filter of all deleted neighbourhoods of $l$ in $X$. Let $\mathscr{F}$ be the collection of subsets $A \subset N$ so that $A \in \mathscr{F} \Leftrightarrow \exists$ a member $V \in \mathfrak{V}$ so that $A=\{i \mid f(i) \in V$ and $i \in N\}$. Then it is easily seen that $\mathscr{F}$ is a filter on $N$ which is free. So $\mathscr{F}$ is contained in a free ultrafilter $p_{0} \in \beta N$. Let $g: N \cup\left\{p_{0}\right\} \rightarrow X$ be the function so that $g\left(p_{0}\right)=l$ and $g \mid N=f$. Then $g$ is a continuous extension of $f$ to $N \cup\left\{p_{0}\right\}$.

THEOREM 1.14. For every $p \in \beta N-N$ let $Z_{p}$ be a $T_{2}$ topological space which is not p-compact. Then $\Pi_{p \in \beta N-N} Z_{p}$ is not countably compact. Hence there exists a family of sequentially compact, $T_{2}$, locally compact, locally countable, scattered, first countable spaces whose product is not countably compact.

Proof. For every $p \in \beta N-N$ let $f_{p}: N \rightarrow Z_{p}$ be a one-to-one function from $N \rightarrow Z_{p}$ which does not extend continuously to a function $g_{p}: N \cup\{p\}$ $\rightarrow Z_{p}$. Let $f: N \rightarrow \Pi_{p \in \beta N-N} Z_{p}$ be the product function $\Pi_{p \in \beta N-N} f_{p}$ defined by the rule that the $p$ th coordinate of $f(n)$ is $f_{p}(n)$ for all $n \in N$ and $p \in \beta N-$ $N$. Then $f$ is a 1-1 map from $N$ into $\Pi_{p \in \beta N-N} Z_{p}$. Now let $p_{0} \in \beta N-N$ be given. Then $f$ cannot extend continuously to a function $\phi: N \cup\left\{p_{0}\right\} \rightarrow$ $\prod_{p \in \beta N-N} Z_{p}$. For if such a $\phi$ exists then the function $g=\phi_{p_{0}}{ }^{\circ} \phi: \Pi_{p \in \beta N-N} \rightarrow$ $Z_{p_{0}}$ will be a continuous extension of $f_{p_{0}}$ to a function from $\prod_{p \in \beta N-N} \rightarrow Z_{p_{0}}$ where $\phi_{p_{0}}$ is the projection from $\Pi_{p \in \beta N-N} Z_{p}$ onto $Z_{p_{0}}$. So $f(N)$ will be an infinite set which will have no cluster point in $\Pi_{p \in \beta N-N} Z_{p}$ by Lemma 1.13. So $\Pi_{p \in \beta N-N} Z_{p}$ is not countably compact.

Now taking $Z_{p}=X_{p}$ of Lemma 1.12 for each $p \in \beta N-N$ we get the last assertion of our theorem.

REMARK. The V-process of M. Rajagopalan is very effective in solving 
some problems in topology considered by M. E. Rudin [10], A. V. Arhangel'skii [1] and others on constructing hereditarily separable, regular, nonLindelöf spaces and compact $c$-spaces. These results obtained by the first author will appear elsewhere.

K. Kunen, and Eric Van Douwen informed the authors in a private communication that by a different method they also got spaces like $X_{p}$ of Definition 1.8. Recently their technique was also used by J. E. Vaughn [12] to get the same type of spaces. Both J. E. Vaughn and K. Kunen use (CH). Eric Van Douwen uses an axiom weaker then $(\mathrm{CH})$. Their results are very recent and the results of this paper were obtained in 1974 and were announced in [13]. Recently M. Rajagopalan got the solution to the above-mentioned problem of C. Scarborough and A. H. Stone using an axiom called $\circledast$ which is weaker than both (CH) and (MA) [14].

The following result is of independent interest.

THEOREM 1.15. There exists a completely regular $T_{2}$ sequentially compact space $M$ which is not $p$-compact for any $p \in \beta N-N$. Then $M^{2^{c}}$ is not countably compact.

Proof. Let $x_{0}$ be a fixed element of $\Pi_{p \in \beta N-N} X_{p}$ where $X_{p}$ is the space constructed by V-process above for each $p \in \beta N-N$. Let $M$ be the set of all elements of $\Pi_{p \in \beta N-N} X_{p}$ which differ from $x_{0}$ in at most a countable number of coordinates. Then $M$ is easily seen to be sequentially compact. Now $M$ contains a closed subspace homeomorphic to $X_{p}$ whatever $p \in \beta N-N$ may be. Since $X_{p}$ is not $p$-compact, it follows that $M$ is not $p$-compact where $p \in \beta N-N$. Let us put $M_{p}=M$ for all $p \in \beta N-N$. Then imitating the proof of Theorem 1.14 we get that $\Pi_{p \in \beta N-N} M_{p}=M^{2^{c}}$ is not countably compact.

\section{REFERENCES}

1. A. V. Arhangel'skii, On cardinal invariants, General Topology and its Relations to Modern Analysis and Algebra III (Proc. Third Prague Topological Sympos., 1971), Academia, Prague; Academic Press, New York, 1972, pp. 37-46. MR 49 \#3800.

2. A. R. Bernstein, $A$ new kind of compactness for topological spaces, Fund. Math. 66 (1969/70), 185-193. MR 40 \#4924.

3. J. Dugundji, Topology, Allyn and Bacon, Boston, Mass.,1966. MR 33 \# 1824.

4. L. Gillmann and M. Jerison, Rings of continuous functions, Van Nostrand, Princeton, N. J., 1960. MR 22 \#6994.

5. V. Kannan and M. Rajagopalan, On scattered spaces, Proc. Amer. Math. Soc. 43 (1974), 402-408. MR 48 \# 12469.

6. __ Scattered spaces. II, Illinois J. Math. (to appear).

7. M. Rajagopalan, Scattered spaces. III, J. Indian Math. Soc. 41 (1977).

8.,$\beta N-N-\{p\}$ is not normal, J. Indian Math. Soc. (N. S.) 36 (1972), 173-176. MR 47 \#9545.

9. $117-125$. , A chain compact space which is not strongly scattered, Israel J. Math. 23 (1970), 
10. M. E. Rudin, Lectures on set theoretic topology, CBMS Regional Conf. Ser. in Math., no. 23. Amer. Math. Soc., Providence, R. I., 1975. MR 51 \# 4128.

11. C. T. Scarborough and A. H. Stone, Products of nearly compact spaces, Trans. Amer. Math. Soc. 124 (1966), 131-147. MR 34 \#3528.

12. J. E. Vaughn, Products of perfectly normal sequentially compact spaces (to appear).

13. M. Rajagopalan and R. Grant Woods, Products of sequentially compact spaces, Notices Amer. Math. Soc. 22 (1975), A-333. Abstract \# 75T-G29.

14. M. Rajagopalan, Some outstanding problems in topology and the V-process (Proc. Mannheim Conf. in Topology, 1975), Lecture Notes in Math., vol. 540, Springer-Verlag, Berlin and New York,1976, pp. 500-517.

Department of Mathematics, University of Manitoba, Winnipeg R3T 2N2, Manttoba, Canada (Current address of R. G. Woods)

Current address (M. Rajagopalan): Departamento de Matematicas, Universidad de Los Andes, Merida, Venezuela 